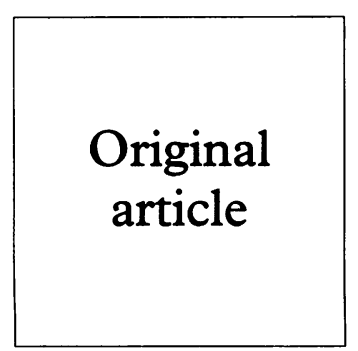

\section{Serological and clinical correlates of gonorrhoea and syphilis in fertile and infertile Nigerian women}

\author{
F E Okonofua, R C Snow, G A Alemnji, A Okoruwa, C O Ijaware
}

Objective: To test the hypothesis that infertile Nigerian women have higher serum levels of $\underset{\vec{\rho}}{\vec{\rho}}$ antibodies against Neisseria gonorrhoea and Treponema pallidum compared with fertile controls. Design: The prevalence of serum antibodies against $N$ gonorrhoea and $T$ pallidum was compared in fertile and infertile Nigerian women.

Setting: Population based case-control study in Ile-Ife, southwestern Nigeria.

Subjects: 60 women with infertility identified from a community based questionnaire survey of 1075 women were compared with 53 age matched fertile controls.

Methods: Sera of fertile and infertile women were tested for the presence of gonococcal antibodies with indirect agglutination test and syphilis antibodies using rapid reagin method.

Main outcome measures: Prevalence of anti-gonococcal and anti-treponemal antibodies in cases and controls. Frequency of self reports of sexually transmitted infections (STIs) in cases and controls.

Results: In comparison with fertile women, infertile women were more likely to report having had repeated lower abdominal pains $(p<0.01)$, yellow vaginal discharge $(p<0.004)$, and whitish vaginal discharge $(p<0.02)$. There was no significant difference between cases and controls in the $\frac{\rho}{2}$ proportions reporting previous STI diagnoses. However, two infertile women reported previous $\vec{c}$ gonococcal infection compared with none in the fertile group. Sixteen of the infertile women $\subsetneq$ $(26.7 \%)$ demonstrated anti-gonococcal antibodies in their sera compared with only four of the 53 व fertile controls $(7.5 \%)(p<0.02$; OR 4.5$)$. There was no significant difference between fertile $\notin$ and infertile women in the proportion showing serological reactivity to $T$ pallidum.

Conclusion: Infertile women have a higher prevalence of anti-gonococcal antibodies compared with fertile controls. Infertile women are also more likely to report previous lower abdominal pains and vaginal discharge. These results provide credible evidence implicating STIs and $N$ 五 gonorrhoea in particular as important factors contributing to female infertility in this population. Public health measures are warranted to address the high rate of STIs and $N$ gonorrhoea in Nigeria.

(Genitourin Med 1997;73:194-197)

Women's Health and Action Research Centre, Obafemi Awolowo University, Ile-Ife, Nigeria

F E Okonofua

R C Snow

G A Alemnji

A Okoruwa

Department of Population Sciences,

Harvard School of

Public Health, Boston,

USA

R C Snow

Department of Chemical Pathology,

Obafemi Awolowo

University, Ile-Ife,

Nigeria

G A Alemnji

Department of

Microbiology and

Parasitology, Obafemi

Awolowo University

Hospital, Ile-Ife,

Nigeria

C O Ijaware

Correspondence to:

Professor F E Okonofua

Director, Women's Health

and Action Research Centre,

Department of Obstetrics

and Gynaecology, Obafemi

Awolowo University, Ile-Ife,

Nigeria.

Accepted for publication

3 February 1997

Keywords: Neisseria gonorrhoea; Treponema pallidum; serology; Nigerian women; infertility

\section{Introduction}

Several studies have documented high rates of infertility in many parts of sub-Saharan Africa. ${ }^{1-3}$ In Nigeria, infertility rates as high as $30 \%$ have been reported in some areas. ${ }^{4}$ Our recent population based study of infertility revealed a secondary infertility rate of $19.7 \%$ among ever married women aged 15-49 years in southwestern Nigeria. ${ }^{5}$ Available evidence indicates that many of the infertility cases in sub-Saharan Africa are attributable to chronic pelvic inflammatory disease. A multicentre study conducted by the World Health Organisation in centres throughout the developed and developing world indicated that $64 \%$ of female patients in African centres had infertility that could be traced to previous pelvic infection. ${ }^{6}$ Hospital based studies in Nigeria report that up to $60 \%$ of infertile women in Nigeria have laparoscopic evidence of previous pelvic infection..$^{78}$ Despite the evidence associating pelvic infection with the high rate of infertility in Africa, there has as yet been little empirical data conclusively to implicate specific pelvic infections or to measure the associ- ation of the known primary causes of pelvic infection with secondary infertility.

Pelvic infections may be due to sexually $\frac{T}{0}$ transmitted diseases or to post-abortal and postpartum infections. Of these, those due to sexually transmitted diseases may be more difficult to relate to infertility since many of the infections that produce tubal damage tend to be asymptomatic. Thus, many studies that have investigated the association between sexu- $\stackrel{\infty}{\rightarrow}$ ally transmitted diseases and infertility have $T$ used self reports of previous pelvic infections, ${ }^{9}{ }^{\circ}$ examination of clinical notes, ${ }^{10}$ laparoscopic evidence of salpingitis, ${ }^{11}$ and serological corre- $\frac{\mathbb{D}}{2}$ lation..$^{1213}$ Of these, serological evidence may be more accurate since it can detect exposure 8 to symptomatic as well as asymptomatic pelvic infections.

Neisseria gonorrhoea and Chlamydia trachomatis are the two sexually transmitted diseases most likely to produce pelvic infection and secondary infertility. Earlier studies have reported higher rates of genital tract infection with $N$ gonorrhoea among infertile Nigerian women compared with fertile pregnant con- 
trols. ${ }^{14}$ Our recent study revealed a significantly higher rate of isolation of $N$ gonorrhoea from the endocervices of infertile women in Ile-Ife compared with pregnant controls. ${ }^{15} \mathrm{~A}$ critical finding in that study was the demonstration of a high rate of isolation of $N$ gonorrhoea among the women, indicating that gonococcal infection is still a predominant public health problem in this population.

Although it is clear that infertile women in this population are more likely than fertile women to demonstrate cervical carriage of $N$ gonorrhoea, there are as yet no data to compare and quantify the level of exposure to the organism in the two groups of women. Such data will be more relevant in determining and measuring the association between infection with $N$ gonorrhoea and infertility in this population. Among Gambian women, Mabey et al ${ }^{13}$ reported higher levels of anti-gonococcal antibodies in women with tubal infertility compared with pregnant controls, providing strong evidence for gonococcal infection as a cause of tubal infertility in the Gambia.

The primary objective of this paper was to report the results of gonococcal serological studies undertaken in infertile and fertile Nigerian women with a view to elucidating the role of $N$ gonorrhoea as a cause of infectious infertility in this population. The results of treponemal serology are also reported to allow better understanding and comparison of the background rate of other sexually transmitted diseases in the two groups of women.

\section{Subjects and methods}

The study was conducted in Ife Central Local Government Area (LGA) of southwestern Nigeria between March 1994 and May 1995. The study drew on population based data to examine a subpopulation of cases and controls. The cases were women with primary and secondary infertility in the LGA who were identified through a comprehensive community survey of the area in 1994. The details of the community survey have been described elsewhere. ${ }^{16}$ In brief, 1075 ever married women aged 15 to 49 years in the area were randomly selected and interviewed in their households with a detailed structured and pretested questionnaire. The questionnaire solicited information on the sociodemographic profiles of the women and their pregnancy and contraception histories. Inquiries were also sought into their gynaecological histories with particular reference to their previous experience of symptoms consistent with sexually transmitted infections. Specifically, the women were asked a series of questions as to whether they currently had or had ever had symptoms suggesting an STI or had ever been diagnosed as having a specific STI. The symptoms checklist included repeated lower abdominal pain, yellow vaginal discharge, whitish vaginal discharge, repeated itching in the genital area, repeated painful/burning sensation on micturition, and ulcers in the genital area.

After the survey, women were classified by fertility status based on an algorithm designed for the purpose by the World Health Organisation. ${ }^{17}$ Women classified as suffering from primary and secondary infertility formed the cases for this study. The controls were women who were classified as being fertile, and they were matched within 2 years by age and resident locality to the infertile cases. Six women in the control group were pregnant at the time of the survey. Both cases and controls were revisited within 1 month of the initial survey in their households to obtain blood samples for estimating gonococcal and treponemal antibodies. The purpose of the study was fully explained to the women and only those who agreed to participate were finally included into the study. The protocol for the study was approved by the ethics review committees of the Obafemi Awolowo University and the Harvard School of Public Health, Boston, USA. The interviews were conducted by trained female interviewers. Women were assured of confidentiality of information and of the results of the blood samples.

A volume of $15 \mathrm{ml}$ of blood was taken from the women in plain tubes, allowed to clot for 2 hours, centrifuged at $3000 \mathrm{rpm}$ for $15 \mathrm{~min}$ utes, and separated into sera. Sera were refrigerated at $-20^{\circ} \mathrm{C}$ until assayed in batches for antibodies. The presence of gonorrhoea antibodies was determined with a gonorrhoea agglutinotest kit (Istituto Immunologico Italiano, Pomezia, Rome) made up by a stabilised suspension of polystyrene latex particles on which an antigenic extract of strains of $N$ gonorrhoea in smooth stage is absorbed. Detection of antibody to pilin proteins has been reported as being valid in diagnosing current and previous gonococcal infection. ${ }^{1819}$ Serological testing for syphilis was carried out with the rapid plasma reagin method as described by the World Health Organisation. ${ }^{20}$

For statistical analysis, the proportion of infertile and fertile women demonstrating gonococcal and treponemal antibodies in their sera was determined. The prevalence of symptoms of STIs and previous specific STI diagnoses was also determined in both groups. These variables were then compared using $\chi^{2}$ test with Yates's correction.

\section{Results}

In the population based study, 208 women in the sample of 1075 women $(19 \cdot 4 \%)$ were classified as having primary and secondary infertility in Ile-Ife. During subsequent follow up, 84 cases and 75 age matched controls were found at home. However, only 60 infertile and 53 fertile women who were approached agreed to participate in the study. Therefore, 60 infertile women and 53 fertile controls met the criteria for inclusion into the study. Among the infertile cases, there were no significant differences between responders and non-responders in mean age, sociodemographic characteristics, and type of infertility. However, non-responders had slightly longer mean duration of infertility compared with responders $(7 \cdot 5$ years in non-responders versus 6.0 years in respon- 
Comparison of the distribution of symptoms of sexually transmitted infections (STIs) in infertile women with fertile controls

\begin{tabular}{|c|c|c|c|c|c|}
\hline & $\begin{array}{l}\text { Infertile } \\
n(\%)\end{array}$ & $\begin{array}{l}\text { Fertile } \\
n(\%)\end{array}$ & Odds ratio & p Value & $C I$ \\
\hline Number & 60 & 53 & & & \\
\hline No symptoms & $12(20 \cdot 0)$ & $32(60 \cdot 4)$ & $0 \cdot 2$ & 0.000 & $0 \cdot 1-0.4$ \\
\hline Repeated lower abdominal pain & $28(46 \cdot 7)$ & $12(22 \cdot 6)$ & $3 \cdot 0$ & 0.01 & $1 \cdot 2-7 \cdot 4$ \\
\hline Yellow vaginal discharge & $25(41 \cdot 7)$ & $8(15 \cdot 1)$ & $4 \cdot 0$ & 0.004 & $1 \cdot 5-11 \cdot 1$ \\
\hline Whitish vaginal discharge & $30(50 \cdot 0)$ & $11(20.8)$ & $3 \cdot 8$ & 0.002 & $1 \cdot 5-9 \cdot 6$ \\
\hline Repeated itching in the genital area & $30(50 \cdot 0)$ & $18(34.0)$ & 1.9 & NS & $0.9-4.5$ \\
\hline Repeated painful/burning sensation on micturition & $10(16 \cdot 7)$ & $3(5 \cdot 7)$ & $3 \cdot 3$ & NS & $0 \cdot 8-16 \cdot 3$ \\
\hline Ulcer in the genital area & $2(3 \cdot 3)$ & $0(0 \cdot 0)$ & - & - & - \\
\hline Previous diagnosis of a specific STI & $6(10 \cdot 0)$ & $1(1 \cdot 9)$ & $5 \cdot 8$ & NS & $0 \cdot 7-131 \cdot 8$ \\
\hline
\end{tabular}

ders). Among the infertile women, 59 had secondary infertility while only one had primary infertility. The duration of infertility ranged from 2 to 17 years, with a median of 7 years. Both cases and controls were of similar age and educational status and an equal proportion of the women were resident in the urban and rural areas of the locality.

The results of the distribution of reported symptoms of STIs in cases and controls are presented in the table. Consistent with findings from the total population sample of 1075,5 infertile cases were significantly more likely than fertile controls to report having had repeated lower abdominal pains, yellow vaginal discharge, and whitish vaginal discharge. By contrast, there was no statistical difference between cases and controls in the proportion reporting repeated itching in the genital area, repeated painful sensation on micturition, and ulcer in the genital area. There was also no difference between both groups in the proportion reporting specific STI diagnoses. Out of six infertile women reporting specific STI diagnoses, two reported candidiasis, two trichomoniasis, while two reported having had gonorrhoea. The only woman reporting specific STI in the fertile group admitted having candidiasis. None of the women in the two groups reported infection with syphilis or other STI diagnoses. There were no differences between cases and controls in the number of reported abortions (three in cases and one in controls). Thus, after controlling for number of reported abortions and women's age, the pattern of observed differences in reported symptoms of previous STIs between cases and controls remained.

Regarding the serological results, 16 of the 60 infertile women $(26 \cdot 7 \%)$ demonstrated gonococcal antibodies in their sera compared with only four of the 53 fertile controls $(7 \cdot 5 \%)$. This difference was statistically significant $\left(\chi^{2}\right.$ test $=5.8 ; p<0.02$; odds ratio (OR) $4 \cdot 5, \mathrm{CI}=1 \cdot 3-19 \cdot 9)$. By contrast, there was no significant difference between cases and controls in the proportions showing positive reactivity to treponemal antibodies. Six infertile women $(10.0 \%)$ compared with nine fertile controls $(17 \cdot 0 \%)$ had positive reactivity to treponemal antibodies, a difference that was not statistically significant $\left(\chi^{2}\right.$ test $=0.7 ; \mathrm{p}>0.5$; $\mathrm{OR}=0 \cdot 5, \mathrm{CI}=0 \cdot 2-1 \cdot 8)$.

\section{Discussion}

Several STIs have been implicated as possible aetiological agents in female infertility in developing countries, notably Neisseria gonorrhoea, Chlamydia, and bacterial vaginosis. ${ }^{1}$ As $\stackrel{5}{+}$ their relative contribution to female infertility may vary in different populations, it is important that inferential approaches are used to measure their importance in communities where the prevalence of infertility is known to be high.

The results of this study indicate that a sig- $\overrightarrow{\vec{\omega}}$ nificantly higher proportion of infertile women in southwestern Nigeria have serological evi- $\stackrel{\rho}{=}$ dence of previous exposure to $N$ gonorrhoea $\omega$ compared with fertile women. Infertile women $\omega$ were also more likely to report having had $\vec{\oplus}$ lower abdominal pains, vaginal discharge, and 의 previous gonococcal infection. The results of this study and that of our earlier study which $c$ demonstrated higher rates of isolation of $N \stackrel{\bar{D}}{\sigma}$ gonorrhoea in the lower genital tract of infertile women compared with fertile controls ${ }^{15}$ pro- 8 vide evidence that $N$ gonorrhoea may be an important precursor of infertility among Nigerian women in this region. The results with treponemal antibodies did not show any significant differences between infertile cases $\stackrel{\mathbb{D}}{\stackrel{2}{2}}$ and fertile controls. This is not surprising $\overrightarrow{\hat{0}}$ since syphilis is not known to be a sterilising STI; however, this result does provide evidence for an increasing and persisting high rate of both syphilis and $N$ gonorrhoea in this $\stackrel{\infty}{=}$ population. The finding that several women $\frac{5}{3}$ with treponema antibodies had no history of genital ulcer reflects the inadequacy of historical evidence in confirming previous exposure to STIs. However, this does not invalidate our results since the tendency applies equally well to cases as well as controls.

The use of serology may underestimate the true extent of gonococcal infection since anti- $\tilde{N}$ bodies tend to disappear with time. W Nevertheless, for an infection that is often asymptomatic, and in a community where $\stackrel{\varrho}{c}$ routine testing for STIs is not the norm, serology provides the best approach to study the association between gonococcal infection and infertility. In particular, since the study was population based, serology had the additional $\stackrel{\mathbb{D}}{\triangle}$ advantages of reduced costs, feasibility, and acceptability when used in the local community.

The results of this study suggest that secondarily infertile women in southwest Nigeria? have four times the rate of previous exposure to $N$ gonorrhoea compared with fertile women. Pelvic infection with $N$ gonorrhoea leads to infertility as a result of the development of bilateral tubal obstruction and/or pelvic adhesions. ${ }^{1}$ Direct evidence of gonococcal involve- 
ment in tubal damage can be found in studies which compare rates of past infection with $N$ gonorrhoea in cases of tubal infertility versus non-tubal infertility. ${ }^{1213}$ Recent studies $^{21-23}$ report continuing high prevalence of symptomatic and asymptomatic gonococcal infection among different groups of Nigerian women. The infections have been attributed to the fragile nature of the health infrastructure, deteriorating socioeconomic conditions, and harmful traditional beliefs and practices. ${ }^{24}$ The results of several qualitative studies ${ }^{19} 20$ indicate that, owing to wrong perceptions about the causes of the illness, many STIs in Nigerian women are poorly treated and are often attended with delayed treatment. Such delay in the treatment of gonococcal infection has been reported to increase the likelihood that affected women would develop post-infectious infertility. ${ }^{25}$ Clearly, culturally appropriate educational programmes to increase awareness of the causes, dangers, and ways to prevent and treat STIs, including $N$ gonorrhoea, would be crucial measures to reduce the burden of infertility in this population.

In conclusion, the results of this population based case-control study indicate that STIs are significantly associated with infertility among Nigerian women. In particular, women with secondary infertility have four times the rate of previous exposure to $N$ gonorrhoea compared with fertile women. Further studies are warranted to measure the contribution of other sterilising STIs-Chlamydia and bacteria vaginosis-to female infertility in this population. Public health measures directed at reducing the burden of STIs will be crucial in preventing infertility in Nigeria.

We are grateful to Olukemi Orioke, Ufuoma Eruotor, Helen Ajabor, Oye John, and Yinka Abulude for their support and assistance at various stages of this study.

The study was carried out with a grant from the Ford Foundation, grant number 930-0988. We thank Dr Natalia Kanem, Ford Foundation's representative in West Africa for her support and encouragement throughout the period of the study.

1 Sciarra J. Infertility: an international health problem. Int $\mathcal{F}$ Gynaecol Obstet 1994;46:155-63.

2 Cates W, Farley TMM, Rowe PJ. Worldwide patterns of infertility: is Africa different? Lancet 1985;ii:596-8.

3 Belsey MA. The epidemiology of infertility: a review with particular reference to sub-Saharan Africa. Bull World Health Organ 1976:319-41.

4 Adetoro OO, Ebomoyi EW. The prevalence of infertility in a rural Nigerian community. Afr f Med Med Sci 1991; 20:23-7.

5 Snow RC, Okonofua FE, Kane T, Farley TMM, Pinol A. Prevalence and determinants of infertility in Ile-Ife, Nigeria. Contracept Fertil Sex Special No 1. Suppl no 9, 1995;23:S54.

6 World Health Organisation. Infections, pregnancies and infertility: perspectives on prevention. Fertil Steril 1987; 47:964.

7 Otolorin EO, Ojengbede O, Falase OA. Laparoscopic evaluation of the tuboperitoneal factors in infertile Nigerian women. Int $\mathcal{F}$ Gynecol Obstet 1987;25:47.

8 Okonofua FE, Esen UI, Nimalaraj T. Hysterosalpingography versus laparoscopy in tubal infertility:
comparisons based on findings at laparotomy. Int $\mathcal{f}$ Gymecol Obstet 1989;28:143.

9 Sherman KJ, Daling JR, Weiss NS. Sexually transmitted diseases and tubal infertility. Sex Transm Dis 1987; 14:12-6

10 Nylander PPS, Ladipo OA. Infertility status in rural Nigeria. World Health Organisation Commun Surv 1979.

11 Faro S. Infection and infertility. Infect Dis Obstet Gynaecol 1993;1:51.

12 Tiiam KH, Zeilmaker GH, Alberda AT, evan Heijst BY, de Roo JC, Polak-Vogelzang AA, et al. Prevalence of antibodies to Chlamydia trachomatis, Neisseria gonorrhoea, and Mycoplasma hominis in infertile women. Genitourin and Mycoplasma hom $1985 ; 61: 175-8$.

13 Mabey DCW, Ogbaselassie G, Robertson JN, Heckels JF, Ward ME. Tubal infertility in the Gambia: chlamydial and gonococcal serology in women with tubal occlusion compared with pregnant controls. Bull World Health Organ 1986;63:1107.

14 Onifade A, Osoba OA. Venereal diseases among women complaining of infertility. West Afr 7 Med 1975;23:284-6.

15 Okonofua FE, Ako-Nai KA, Dighitoghi MD. Lower genital tract infections in infertile Nigerian women compared to controls. Genitourin Med 1995;71:163-8.

16 Okonofua FE, Snow RC. Determinants and prevalence of infertility in Ile-Ife, Nigeria. Ford Foundation grant report, Number 930-0988, 1995.

17 World Health Organisation Special Programme of Research, Development and Research Training in Human Reproduction. Task Force on the Prevention and Management of Infertility. Survey of the prevalence of primary and secondary infertility: a core protocol. of primary and second
Geneva: WHO, 1991.

18 Hermodsen MA, Chen KCS, Buchanan TM. Neisseria pili proteins: aminoterminal amino acid sequences and identification of an unusual amino acid. Biochemistry 1978;17: 442-5.

19 Reimann K, Odum L, Larsen SO, Lind I. Indirect haemaglutination test using gonococcal pilus antigen: how useful to diagnose gonorrhoea? Genitourin Med 1987;63:250-5.

20 Van dyck E, Piot P, Meheus A. Bench-level laboratory manual for sexually transmitted diseases. WHO/ VDT/89.443.

21 Olukoya A, Elias C. Perceptions of reproductive morbidity among Nigerian men and women: implications for family planning services. Final Project report. Population Council Contract No C192.20A, 1994.

22 Erwin JO. Reproductive tract infections among women in Ado-Ekiti, Nigeria: symptoms recognition, perceived causes and treatment choices. Health Transition Rev 1993;3:Suppl 135-49.

23 Sogbetun AO, Alausa KO, Osoba OA. Sexually transmitted diseases in Ibadan, Nigeria. Br $\mathcal{F}$ Vener Dis 1982; 53:155-60.

24 Adekunle OA, Ladipo OA. Reproductive tract infections in Nigeria: challenges for a fragile health infrastructure. In: Germain A, Holmes KK, Piot P, Wasserheit JN, eds. Reproductive tract infections. New York: Plenum Press, 297-316.

25 Hillis SD, Joesoef R, Marchbanks PA, Wasserheit JN, Cates W Jr, Westrom L. Delayed care of pelvic inflammatory disease as a risk factor for impaired fertility. $A m \mathcal{F}$ Obstet Gynecol 1993;168:1503. 